\title{
Identifying futility in a paediatric critical care setting: a prospective observational study
}

\author{
A Y Goh, Q Mok
}

\begin{abstract}
Aims-To determine the extent of futile care provided to critically ill children admitted to a paediatric intensive care setting.

Methods-Prospective evaluation of consecutive admissions to a 20 bedded multidisciplinary paediatric intensive care unit of a North London teaching hospital over a nine month period. Three previously defined criteria for futility were used: (1) imminent demise futility (those with a mortality risk greater than $90 \%$ using the Paediatric Risk of Mortality (PRISM II) score); (2) lethal condition futility (those with conditions incompatible with long term survival); and (3) qualitative futility (those with unacceptable quality of life and high morbidity).

Results-A total of 662 children accounting for 3409 patient bed days were studied. Thirty four patients fulfilled at least one of the criteria for futility, and used a total of 104 bed days (3\%). Only $33(0.9 \%)$ bed days were used by patients with mortality risk greater than $90 \%, 60(1.8 \%)$ by patients with poor long term prognosis, and $16(0.5 \%)$ by those with poor quality of life. Nineteen of 34 patients died; withdrawal of treatment was the mode of death in $15(79 \%)$.

Conclusions-Cost containment initiatives focusing on futility in the paediatric intensive care unit setting are unlikely to be successful as only relatively small amounts of resources were used in providing futile care. Paediatricians are recognising futility early and may have taken ethically appropriate measures to limit care that is futile.

(Arch Dis Child 2001;84:265-268)
\end{abstract}

Keywords: cost containment; medical futility; resource consumption

Paediatric Intensive Care Unit, Great Ormond Street Hospital for Children, London WC1N 3JH, UK

A Y Goh

Q Mok

Correspondence to: Dr A Y Goh, Paediatric Intensive Care Unit, Department of Paediatrics, University Malaya Medical Centre, 50603 Kuala

Lumpur, Malaysia

adrian@

medicine.med.um.edu.my

Accepted 4 July 2000 children and their families. Furthermore, recognition that resources are finite has made health managers look at numerous methods of cost containment, including controversial attempts to limit care that is deemed futile. ${ }^{2-4}$ It is well known that the intensive care unit (ICU) is costly and that compared to routine hospital care, ICU bed charges can be $500 \%$ higher $^{5}$ and can consume up to $20 \%$ of total hospital expenditure. ${ }^{67}$

Published research from adult intensive care shows that significant potential cost savings can be achieved by identifying and terminating care that is futile. ${ }^{89}$ There is a tendency to generalise these findings to children as these issues have not been widely investigated in paediatric populations. Sachdeva et al in North America, however found that only relatively small amounts of resources were consumed in futile paediatric ICU (PICU) care. ${ }^{10}$ The reason for this difference between the adult and paediatric ICU population has not been fully studied. In addition, the extent and resource consumption from medical futility has rarely been investigated in a PICU setting outside North America. Recognising the importance of knowing the limits of critical care, we set out to determine the extent of futile care provided in a tertiary PICU in the London area of the UK, using previously defined criteria for futility, and investigate the causes for this discrepancy, if any, from adult studies.

\section{Methods}

A prospective observational study was carried out from 1 October 1998 to 30 June 1999 in the PICU of Great Ormond Street Hospital for Children, which is a 20 bedded, multidisciplinary unit. The aim was to assess the number of patients and number of bed days used by children who met various operational definitions of medical futility. A bed was considered occupied if a child was in the bed at 0800. A minimum stay of eight hours in the PICU was required to be included in the study. Those staying 8-24 hours were considered to have stayed for one day. Bed days were used to determine the extent of futile care provision and as a surrogate for resource consumption. This was part of a study looking at organisation of intensive care services in the North Thames area, which has been submitted for publication elsewhere. ${ }^{11}$ Data were collected daily by one of the authors (AYG) by reviewing the medical charts and the PICU clinical information system (CareVue 4000, Hewlett-Packard). This included admission and daily Paediatric Risk of Mortality (PRISM II) scores, lethal diagnoses, as well as morbidity and causes of mortality, particularly end of life decision making. A resampling of all 24 hour PRISM score values was done by the author to ensure validity of the 
collected data. Reabstraction of data showed an acceptable inter-rater reliability (intraclass correlation coefficient) of 0.90 . As the data collection did not involve any patient intervention, the institutional review board waived the need for informed consent.

DEFINITIONS OF FUTILITY

The following definitions were adapted from previous studies on futility, ${ }^{10}{ }^{12}$ in an attempt to include as many possible conditions that may be considered "futile". These definitions encompass both short term PICU outcomes using prognostication scores that quantify children with significant likelihood for mortality, as well as potential long term outcomes by measuring children with significant morbidity and poor prognosis after PICU discharge.

Imminent demise futility included children with conditions that would result in near certain death despite continued ICU care and was defined as patients with significant likelihood of mortality exceeding $90 \%$ using the PRISM II score. The PRISM II score is a physiological based severity of illness score that uses 14 routinely measured variables, which when combined with the operative status of the patient, predicts the probability of death for the PICU admission. ${ }^{13}$ The PRISM II score is a recalibration of the original PRISM score based on data from patients studied between 1990 and $1992 .{ }^{13}$ Age is no longer a variable and a fixed 24 hour period is used for gathering data. Likelihood of death $=\exp (\mathrm{R}) / 1+\exp$ $(\mathrm{R})$; where $\mathrm{R}=0.2601 \times$ PRISM (24 hour value) $-0.9762 \times$ operative status -5.9751 . Any bed days occupied by patients with admission mortality risk exceeding 90\% were deemed imminently futile. In order to include as wide a number of patients as possible, any additional patient days with mortality risk greater than $90 \%$ during their period of stay were also included.

Lethal condition futility was defined as children with diagnoses or conditions in which long term survival was unlikely. These conditions were modified from those studied in the adult Study to Understand Prognoses and Preferences for Outcomes and Risk of Treatment (SUPPORT) trial. ${ }^{14}$ These included: (1) metastatic malignancy unresponsive to first line therapy and/or requiring second line treatment; (2) history of existing liver failure; (3) history of heart failure which is documented with ejection fraction less than $20 \%$; (4) history of existing respiratory failure requiring endotracheal intubation and home ventilation; (5) acquired immunodeficiency syndrome with CD 4 counts less than 50/ 1 ; and (6) chromosomal or syndromic conditions incompatible with life into the late teens.

Qualitative futility included conditions in which the child has a high level of morbidity; for the purposes of this study it was defined as: (1) persistent vegetative state before admission and during their stay in the PICU; and (2) brain death. The number of bed days for brain death was scored from the moment clinical evidence of brain death was noted. Bed days occupied by patients that fulfilled any of these criteria were considered qualitatively futile care.

\section{Results}

A total of 662 patients were admitted to the PICU during the nine month period, representing 3409 consecutive patient bed days of study. Mean age of the patients was 35.7 (SD 54.7) months (median 9 months) and mean length of stay was 5.2 (8.4) days (median three days). A total of $77.8 \%$ of the patients $(515 / 662)$ required mechanical ventilation. The mean PRISM II score was 11 (median 10). The overall percentage of bed days utilised by patients fulfilling any one of the definitions of futility was for $104(3 \%)$ of 3409 bed days. A total of $34 / 662$ of the patients $(5.1 \%)$ fulfilled at least one of the criteria for futility.

Thirty three $(0.9 \%)$ of the patient bed days met the operational definition for imminent demise futility as measured by PRISM II with a mortality risk exceeding $90 \%$. Eighteen of the bed days were used by eight patients who died; the remaining 15 bed days were by six patients who survived. The majority (27/33) were patient bed days with mortality risks between $90 \%$ and $95 \%$. Only six patient bed days were utilised by patients with daily mortality risks between $96 \%$ and $99 \%$. Sixteen patients satisfying criteria for lethal condition futility used a total of 60 bed days (1.7\%); eight of these died following withdrawal of treatment. These were mainly patients with disseminated malignancy or malignancies unresponsive to first line therapy (table 1). In the qualitative futility group no patient was in a vegetative state before admission to PICU or during their PICU stay and only 16 bed days were occupied by patients fulfilling criteria for brain death, all of whom died.

Fifty one $(7.7 \%)$ of the 662 patients admitted died in PICU; 37 (72\%) of the deaths were associated with withdrawal or withholding of therapy, and 14 died despite aggressive resuscitative efforts. Of the 34 patients who fulfilled any of the criteria for medical futility, 19 $(56 \%)$ died; of these 15 had some form of limitation of treatment (nine withdrawal or withholding therapy, six extubation after fulfilling brain death criteria), and only four died after failed full resuscitative efforts. Patients who fulfilled criteria for futility had a significantly higher mortality rate than

Table 1 Patients who fulfilled criteria for lethal condition futility

\begin{tabular}{lcc}
\hline Condition & $\begin{array}{l}\text { No. of } \\
\text { patients }\end{array}$ & $\begin{array}{l}\text { Bed days } \\
\text { occupied (\%) }\end{array}$ \\
\hline Malignancies & & \\
$\quad$ ALL, post BMT & 2 & $5(8.3)$ \\
$\quad$ AML, post BMT & 3 & $8(13.3)$ \\
Others & 5 & $24(40)$ \\
Chromosomal/syndromes & 4 & $11(18.3)$ \\
AIDS & 1 & $10(16.7)$ \\
Myopathy with RF & 1 & $2(3.4)$ \\
Total & 16 & $60(100)$ \\
\hline
\end{tabular}

ALL, acute lymphoblastic leukaemia; AML, acute myelogenous leukaemia; BMT, bone marrow transplantation; RF, respiratory failure. 
Table 2 Comparison between patients who fulfilled criteria for medical futility and other non-futile patients

\begin{tabular}{lcc}
\hline Characteristics & Futile $(n=34)$ & Non-futile $(n=628)$ \\
\hline Age (mth) & & \\
Length of stay (days) $^{\star}$ & $40.1(59)$ & $35.4(54)$ \\
PRISM $^{\star}$ & $4.6(4.3)$ & $5.1(8.4)$ \\
Mortality (\%) $_{\text {Treatment limitation in non-survivors (\%) }}$ & $20.5(11.7)$ & $10.7(6.7)$ \\
Precipitating cause of admission (\%) & 79 & 5.1 \\
$\quad$ Postoperative & 2.9 & 37.5 \\
Respiratory & 17.7 & 32.2 \\
Sepsis & 38.2 & 30.3 \\
CNS & 23.6 & 9.1 \\
Trauma & 2.9 & 13.8 \\
Others & 14.7 & 4.7 \\
\hline
\end{tabular}

*Values are mean (SD).

PRISM, Paediatric Risk of Mortality Score; CNS, central nervous system.

non-futile patients $(\mathrm{p}<0.013)$. Table 2 shows characteristics of patients who fulfilled criteria for futility and those that did not.

\section{Discussion}

The intensive care unit symbolises the dilemma of modern healthcare-good outcomes can be achieved in the most critically ill, but at a great expense. High and continuously escalating expenditures in the ICU make rigorous economic evaluations necessary to achieve maximum cost effectiveness. ${ }^{15-17}$ Research from adult intensive care has shown potential savings of $\$ 2-5$ million ( $£ 1.2-3.0$ million) per year by identification of care that is futile. ${ }^{8}$ Treating "futile" patients predicted to die in ICU may cost up to $\$ 210423$ ( $£ 129651$ ) per survivor. ${ }^{9}$ While accounting for only $13 \%$ of all patients, they consume up to $32 \%$ of total resources. ${ }^{8}$ Sachdeva et al, however, showed that these potential cost savings might not apply to the paediatric ICU population as only small amounts of resources were expended on futile care. ${ }^{10}$ These issues have not been investigated in a PICU setting outside North America. Using similarly broad definitions for futility we found that relatively few PICU patient bed days were used for futile care. The strength of the current study was that it was prospectively collected and included a proportionally large number of patient bed days. It was also conducted over an extended duration, to account for a potential skew in data caused by seasonal admission trends in PICU. Our findings appear to agree with the conclusion of Sachdeva et al that futile care was not a source of considerable resource consumption in PICU.

The lack of agreement with the findings from the adult ICU population is probably multifactorial. Our study suggests that paediatricians are readily recognising "futile" care early and dealing with it in an ethically appropriate manner. The majority of deaths in the current study involved some form of withholding or withdrawal of therapy, similar to other paediatric studies on end of life decision making. ${ }^{18}{ }^{19} \mathrm{~A}$ combination of worsening severity of illness, poor response to medical therapy, and poor future quality of life were often the reasons for this assumption. It is likely that the practices for treatment limitation in adult ICUs were much more limited during the period when the adult literature on medical futility was published, although recent reports suggest a substantial increase in these practices. ${ }^{20}$ The wide use of treatment limitation in the current study may actually limit the amount of care given to critically ill children, whom might have deteriorated further and gone on to fulfil any one of the broad definitions of medical futility employed. However, restricting care in the interests of cost containment would stretch the limits of ethical behaviour. These decisions should always be made in the "best interest" of the child. ${ }^{18}$

Prognostication systems like the PRISM score used in our study have been shown to reliably predict mortality in the PICU population. ${ }^{21}$ Additionally any other patient days associated with PRISM scores exceeding 90\% were also included. Although values obtained from days other than the admission day have not been previously validated as indicators of mortality, Chang et al have shown that changes in daily individual physiological data were able to predict death accurately in an adult ICU population. ${ }^{2}$ The cut off using mortality risk of greater than $90 \%$ has been described as too low in assessing medical futility ${ }^{10}$ as shown in our study, where $43 \%$ of these children with high mortality risk were able to survive. Improvement in survival probabilities in PICU may necessitate the use of higher values to define futility. These would further decrease the extent of bed days fulfilling the definition of imminent demise futility.

The definition of lethal condition futility captured the greatest number of patient bed days. This definition attempted to identify children that were likely to die from their underlying disease, although not necessarily in the current admission. These were mainly children with malignancies failing first line therapy. In contrast to adult cancer patients failing first line therapy, the outcome in children is better with rescue therapy such as bone marrow transplantation. ${ }^{22} 23$ It could be argued therefore that treating such children does not constitute futile care. The use of these definitions would therefore lead to an overestimation of the extent of futile care provided.

There are several limitations to this study. Firstly, it was carried out in a single institution and might not be representative of the remainder of the UK. The study unit, being a tertiary unit has active ethical discussions among the intensivists, nursing staff, and family, thus potentially limiting the amount of futile care provided to children who are deemed incurable by actively withholding or withdrawal of therapy. Secondly, the process of gatekeeping, where potentially futile cases are not admitted to the PICU may further lessen the estimate of medical futility. Although length of stay or bed days as used in our study has been shown to be a reliable indicator of resource utilisation, ${ }^{24}$ this was not corrected for severity of illness. An ill child who succumbs may use significant resources despite a short PICU stay, thus leading to possible underestimation.

In conclusion, despite using broad definitions that were likely to lead to overestimation of futility, only small amounts of resources were expended on futile care. Generalising potential cost savings from limitation of futile interven- 
tions in adult studies to the paediatric population is unwarranted. Ethically we are reassured that the majority of care provided to critically ill children appears to be appropriate. Paediatricians are recognising care that is futile and have taken steps that are ethically appropriate to limit it. Further potential cost savings to be achieved from identifying and terminating care that is futile would thus be negligible in a paediatric critical care setting.

1 Schneiderman LJ, Jecker NS, Jonsen AR. Medical futility: its meaning and ethical implications. Ann Intern Med 1990;112:949-54.

2 Chang RWS, Jacob S, Lee B, Pace N. Predicting deaths among intensive care unit patients. Crit Care Med 1988;16: 34-42.

3 Rogers J, Fuller HD. Use of daily Acute Physiology and Chronic Health Evaluation (APACHE) II scores to predict individual patient survival. Crit Care Med 1994;22:1402-5.

4 Luce JM, Wachter RM. The ethical appropriateness of using prognostic scoring systems in clinical management. Crit prognostic scoring system
Care Clin 1994;10:229-41.

5 Ridley S, Biggam M, Stone P. A cost-benefit analysis of intensive therapy. Anaesthesia 1993;48:14-19.

6 Russel LB. The role of technology assessment in cost control. In: McNeil BJ, Cravalho EG, eds. Critical issues in medical technology. Boston: Auburn House, 1980:129-38.

7 Kirton OC, Civetta JM, Hudson Civetta J. Cost effectiveness in the intensive care unit. Surg Clin North Am 1996;76:175-200.

8 Esserman L, Belkora J, Lenert L. Potentially ineffective care: a new outcome to assess the limits of critical care. ҰAMA 1995;274:1544-51.

9 Atkinson S, Bihari D, Smithies M, et al. Identification of futility in intensive care. Lancet 1994;344:1203-6.

10 Sachdeva RC, Jefferson LS, Coss-Bu J, Brody BA. Resource consumption and the extent of futile care in a paediatric consumption and the extent of futile care in a paediat
intensive care unit setting. $\mathcal{F}$ Paediatr $1996 ; 128: 742-7$.

11 Goh AYT, Mok Q. Centralization of paediatric intensive care: are critically ill children appropriately referred to a regional centre? Intensive Care Med. Submitted.
12 Halevy A, Neal RC, Brody BA. The low frequency of futility in an adult intensive care unit setting. Arch Intern Med 1996;156:100-4.

13 DiCarlo JV, Zaitseva TA, Khodataleva TV, et al. Comparative assessment of paediatric intensive care in Moscow, the Russian Federation: a prospective multicentre study. Crit Care Med 1996;24:1403-7.

14 Murphy DJ, Knaus WA, Lynn J. Study population in SUPPORT: patients (as defined by disease categories and mortality projections), surrogates and physicians. I Clin Epidemiol 1990;43:11S-28S.

15 Singer M, Myers S, Hall G, Cohen SL, Armstrong RF. The cost of intensive care: a comparison on one unit between 1989 and 1991. Intensive Care Med 1994;20:542-9.

16 Edbroke DL, Stevens VG, Hibbert CL, et al. A new method of accurately identifying costs of individual patients in intensive care: the initial results. Intensive Care Med 1997;23:645-50.

17 Edbroke D, Hibbert C, Ridley S, et al and The Intensive Care Working Group on Costing. The development of a method for comparative costing of individual intensive care units. Anaesthesia 1999;54:110-20.

18 Royal College of Paediatrics and Child Health. Withholding or withdrawing life saving treatment in children. A framework for practice. London: RCPCH, 1997.

19 Goh AYT, Lum LCS, Chan PWK, et al. Withdrawal and limitation of life support in paediatric intensive care. Arch Dis Child 1999;80:424-8.

20 Prendergrast TJ, Claessens MT, Luce JM. A national survey of end of life care for critically ill patients. Am $\mathcal{F}$ Respir Crit Care Med 1998;158:1163-7.

21 Pollack MM, Ruttimann UE, Getson PR. Paediatric Risk of Mortality (PRISM) score. Crit Care Med 1988;16:111016.

22 Chidel MA, Suh JH, Greskovich JF, et al. Treatment outcome for patients with primary non-small cell lung cancer and synchronous brain metastasis. Radiother Oncol Invest 1999;7:313-19.

23 Niethammer D, Klingebiel T, Ebell W, et al. Which children do benefit from bone marrow transplant? The EBMT Paediatric Diseases Working Party. Bone Marrow Transplant 1996;18:43-6.

24 Gemke RJ, Gonsel RJ, McDonnel J, Van Vught AJ. Patient characteristics and resource utilization in paediatric intensive care. Arch Dis Child 1994;71:291-6. 